High-resolution -glucosidase and radical scavenging profiling combined with HPLCHRMS-SPE-NMR for identification of bioactive constituents in crude extract of Pueraria lobata

Liu, Bingrui; Kongstad, Kenneth Thermann; Nyberg, Nils; Qinglei, Sun; Jäger, Anna; Stærk, Dan

Publication date:

2015

Document version

Early version, also known as pre-print

Citation for published version (APA):

Liu, B., Kongstad, K. T., Nyberg, N., Qinglei, S., Jäger, A., \& Stærk, D. (2015). High-resolution -glucosidase and radical scavenging profiling combined with HPLC-HRMS-SPE-NMR for identification of bioactive constituents in crude extract of Pueraria lobata. Poster session presented at 36th Danish NMR Meeting, Lund, Sweden. 


\title{
High-resolution $\alpha$-glucosidase and radical scavenging pro- filing combined with HPLC-HRMS-SPE-NMR for identification of bioactive constituents in crude extract of Pueraria lobata
}

\author{
Bingrui Liu, Kenneth T. Kongstad, Nils T. Nyberg, Sun Qinglei, Anna K. Jäger, and Dan Staerk
}

\section{Abstract:}

This work describes the identification of active constituents in Pueraria lobata root extract by dual high-resolution $\alpha$-glucosidase inhibition ${ }^{1}$ and radical scavenging profiling ${ }^{2}$ combined with HPLC-HRMS-SPE-NMR. ${ }^{3}$ This analytical platform enabled pinpointing of bioactive constituents in HPLC chromatograms direct from crude extracts. Bioactive constituents were cumulatively trapped on SPE cartridges and the structures identified and elucidated by spectral data obtained in the HPLCHRMS-SPE-NMR mode. A total of 24 compounds were identified, and several of these showed radical scavenging activity while two isoflavonoids showed $\alpha$ glucosidase inhibitory activity.

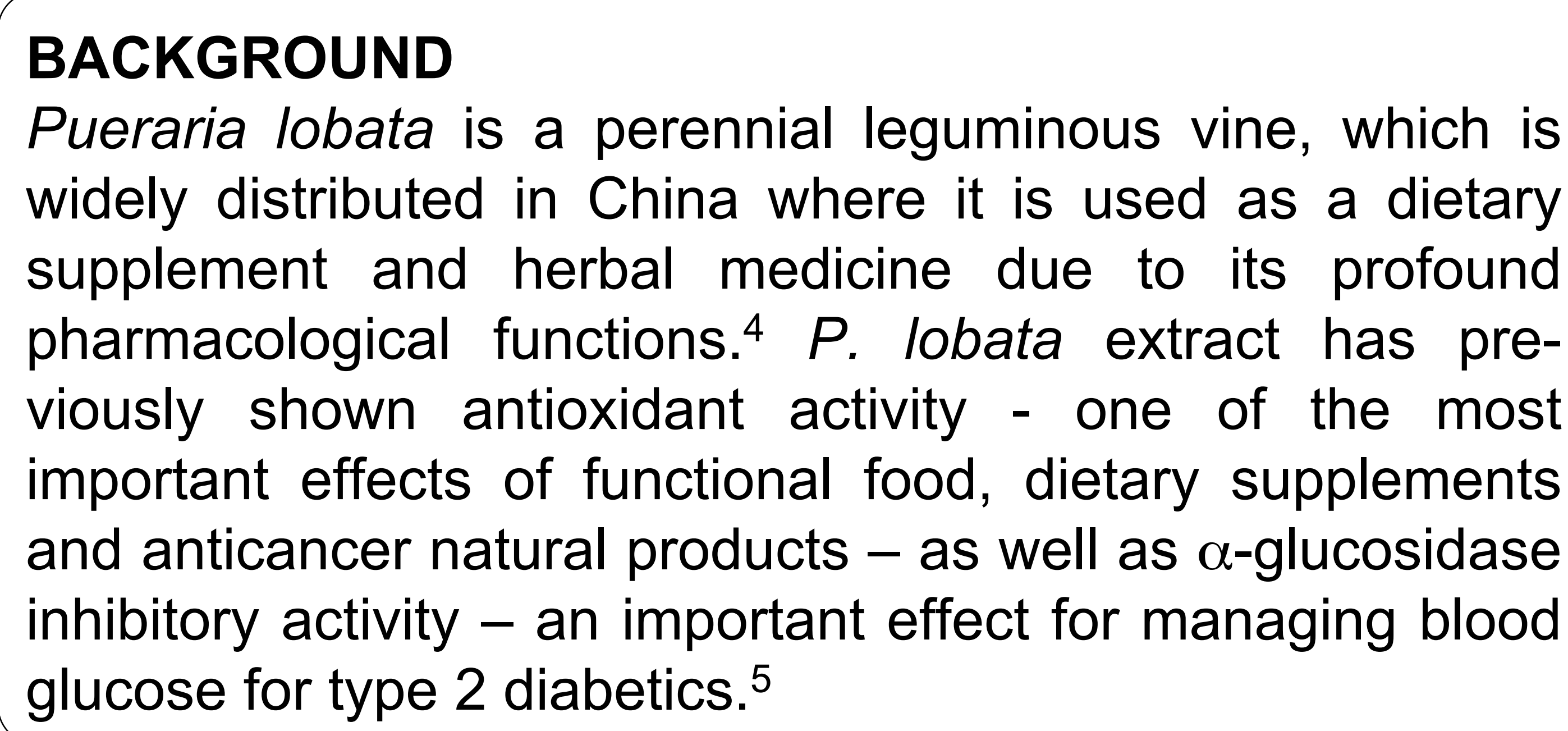

Pueraria lobata is a perennial leguminous vine, which is widely distributed in China where it is used as a dietary supplement and herbal medicine due to its profound pharmacological functions. ${ }^{4} P$. lobata extract has previously shown antioxidant activity - one of the most important effects of functional food, dietary supplements and anticancer natural products - as well as $\alpha$-glucosidase inhibitory activity - an important effect for managing blood glucose for type 2 diabetics. ${ }^{5}$

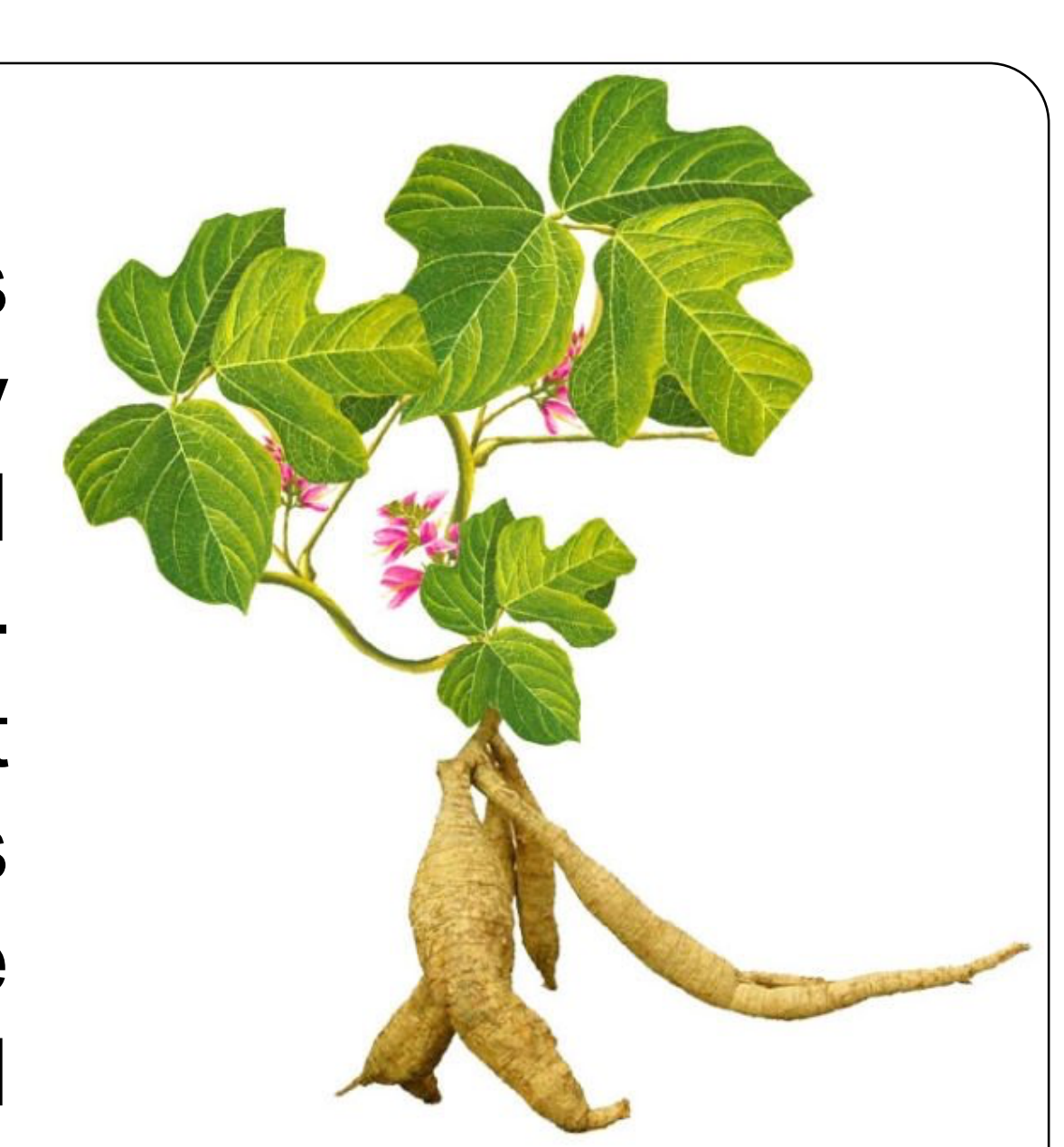

Figure 1. Pueraria lobata

\section{METHOD}

Methanol extract of Pueraria lobata was investigated by dual high-resolution $\alpha$-glucosidase/radical scavenging profiling combined with HPLC-HRMSSPE-NMR.
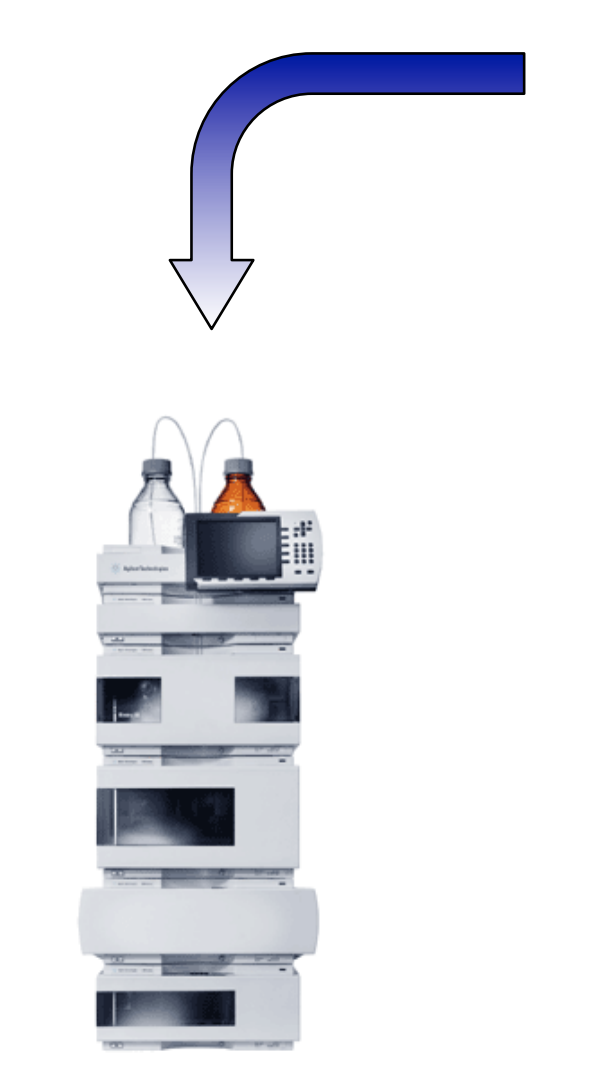

Analytical-scale microfractionation
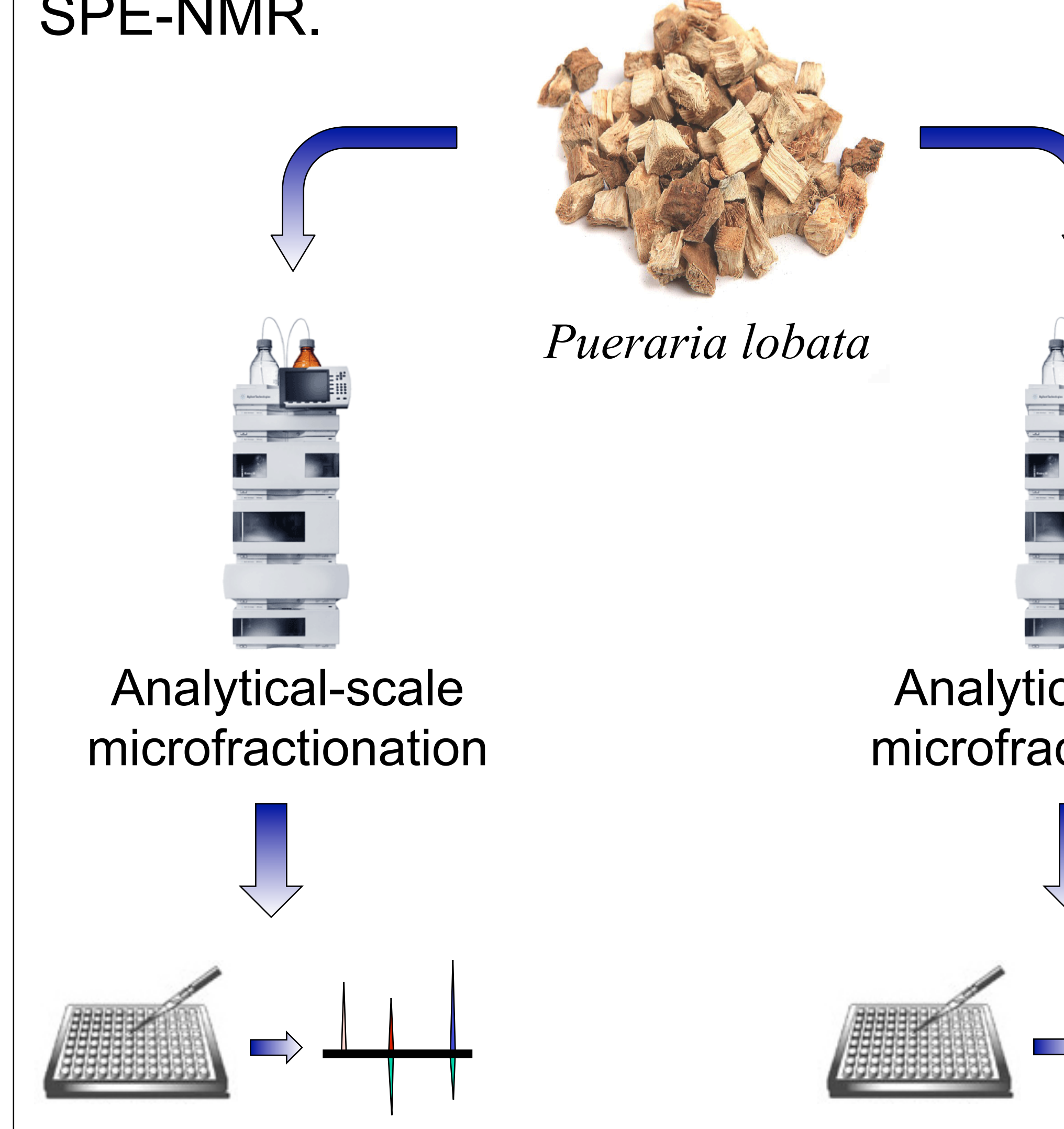

Radical scavenging biochromatogram
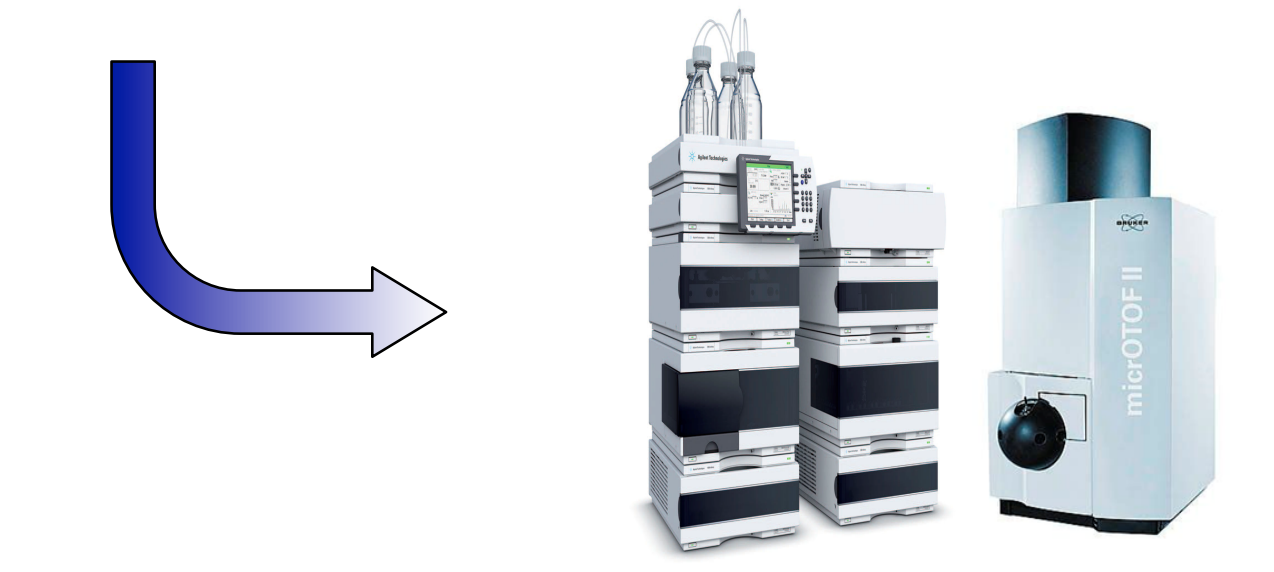

HPLC-HRMS

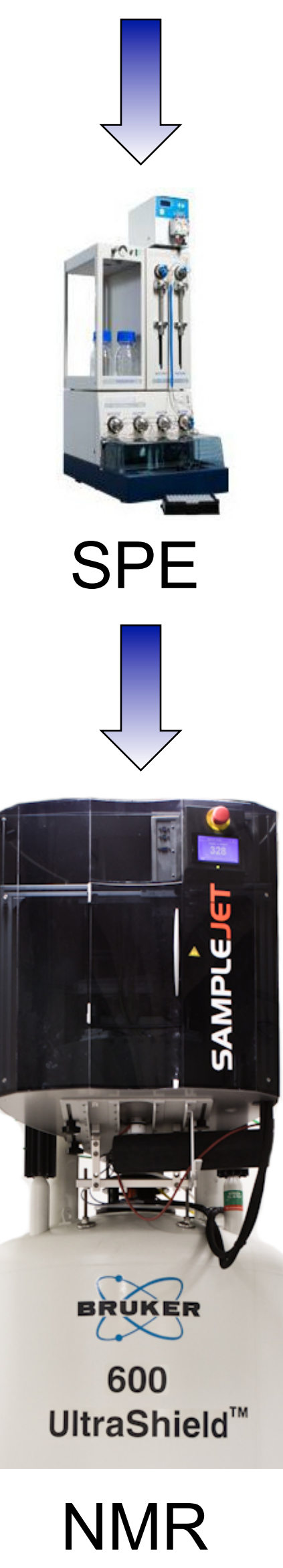

Figure 2. Principle of dual $\alpha$-glucosidase/radical scavenging profiling combined with HPLC-HRMS-SPE-NMR

\section{RESULTS}

Dual high-resolution $\alpha$-glucosidase/radical scavenging profiling provided an $\alpha$-glucosidase inhibition profile (red trace in Figure 3) and a radical scavenging profile (blue trace in Figure 3) below the HPLC chromatogram. This showed several constituents with radical scavenging activity as well as two constituents, i.e., 18 and $\mathbf{2 3}$, with $\alpha$-glucosidase inhibitory activity.

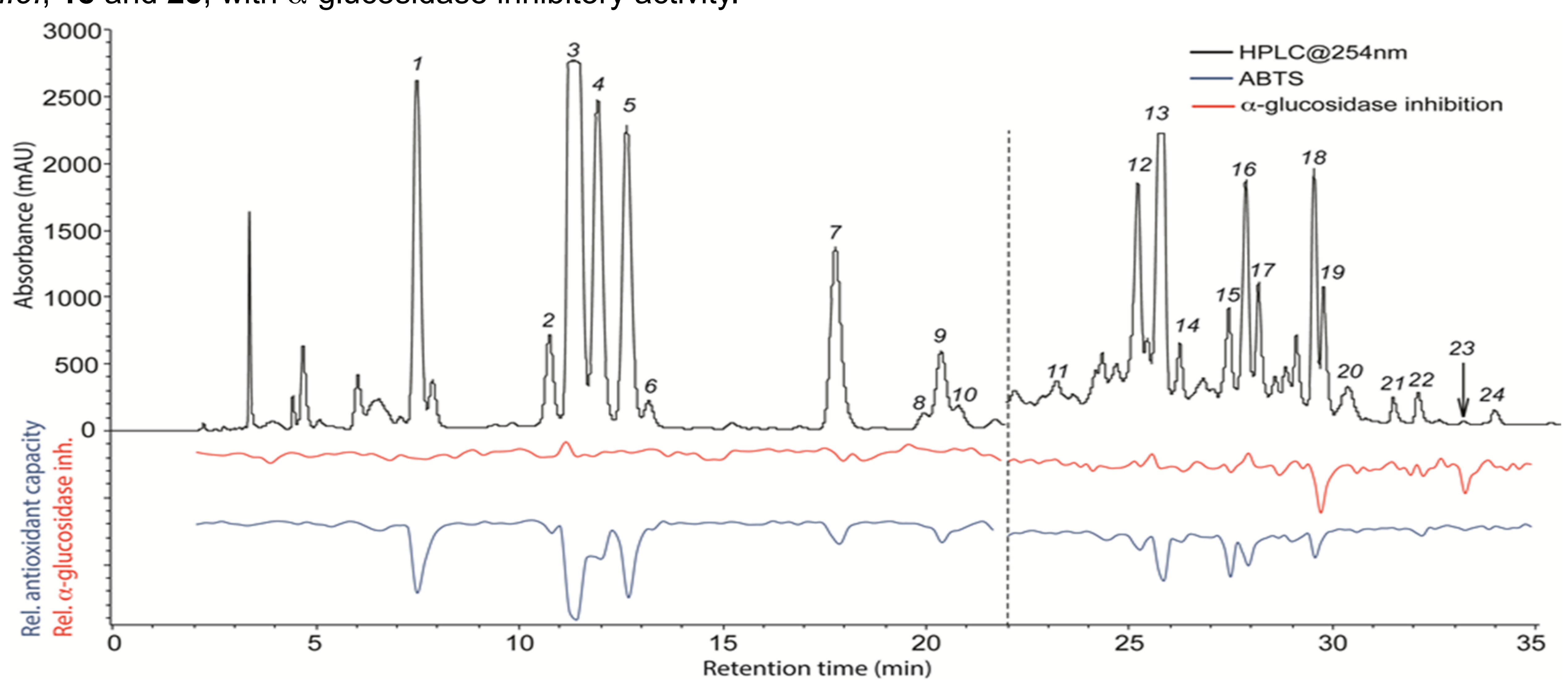

Figure 3. Dual high-resolution $\alpha$-glucosidase inhibition profile and radical scavenging profile.

Analysis of HRMS and NMR data obtained in the HPLC-HRMS-SPE-NMR mode led to identification of 24 compounds (Figure 4), of which $\mathbf{6}$ and 14, were new compounds.

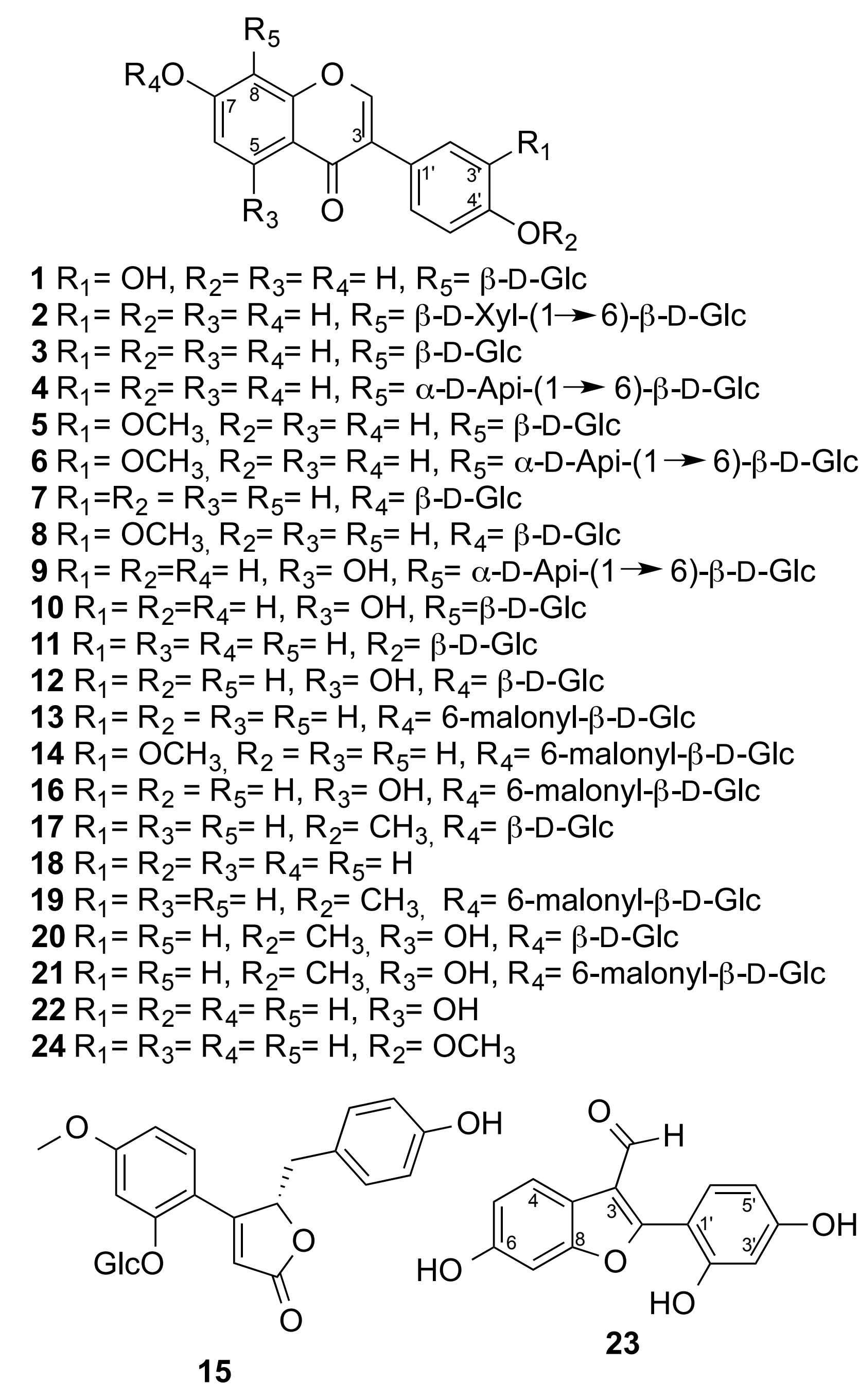

Figure 4. Structure of 1-24

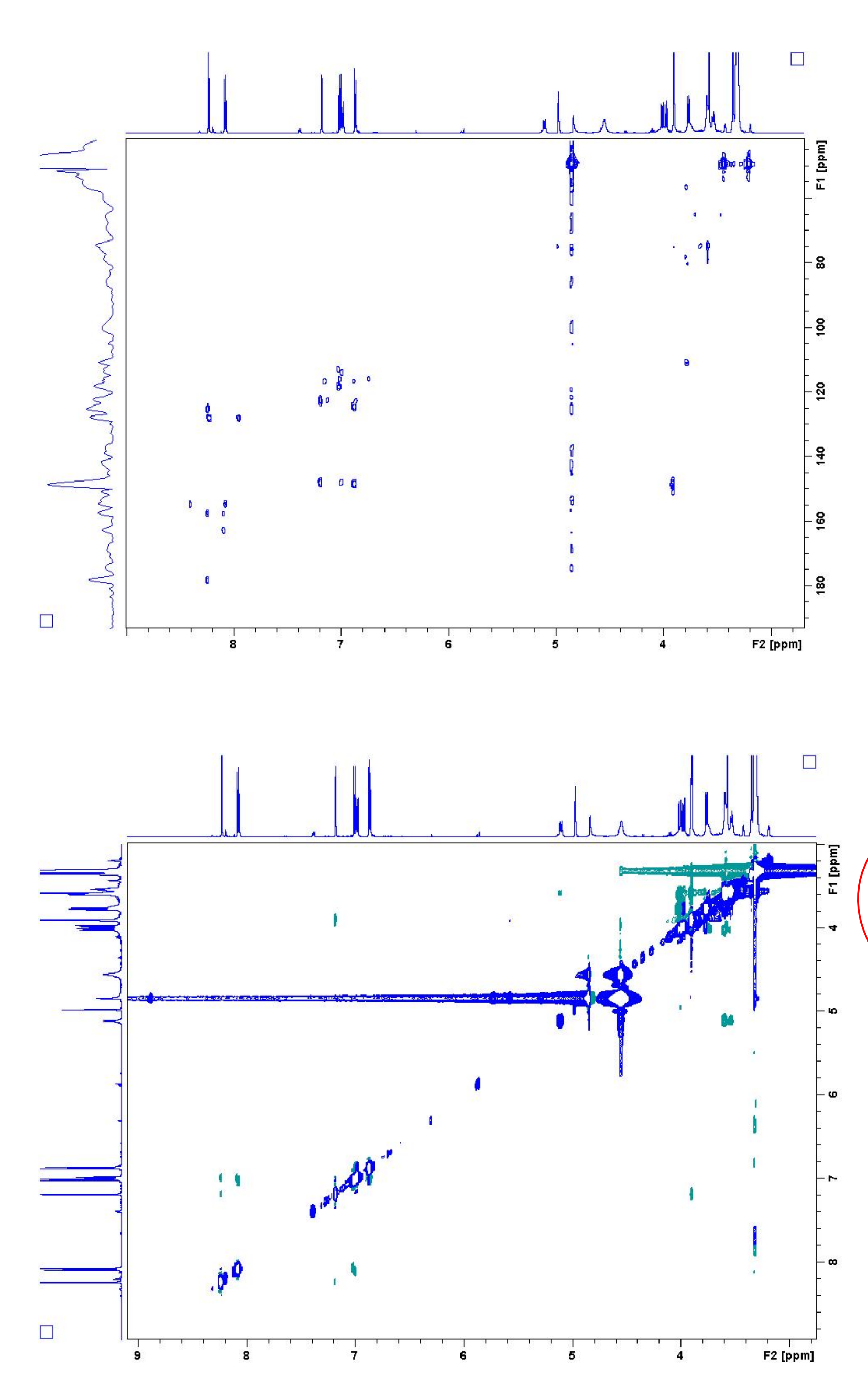

Figure 5. Key HMBC and ROESY correlations in spectra of 6

\section{Conclusion}

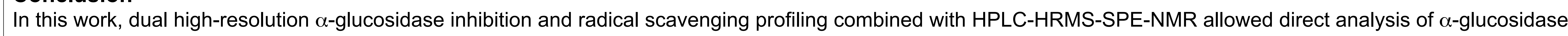

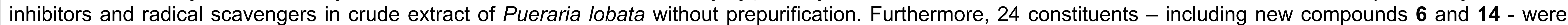
identified by analysis of HRMS and NMR spectral data. This work shows the full advantage of high-resolution bioactivity profiling/HPLC-HRMS-SPE-NMR, and promise even more efficient research in functional food, dietary supplements and traditional medicine.

\section{REFERENCES}

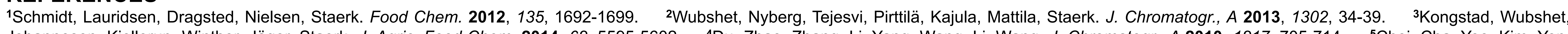

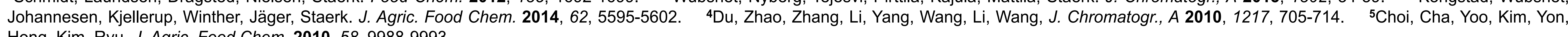
Hong, Kim, Ryu. J. Agric. Food Chem. 2010, 58, 9988-9993. 